\title{
Asian Patients with Stroke plus Atrial Fibrillation and the Dose of Non-Vitamin K Oral Anticoagulants
}

\author{
Oh Young Bang, ${ }^{a}$ Keun-Sik Hong, ${ }^{\mathrm{b}}$ Ji Hoe Heo ${ }^{\mathrm{c}}$ \\ ${ }^{a}$ Department of Neurology, Samsung Medical Center, Sungkyunkwan University School of Medicine, Seoul, Korea \\ ${ }^{b}$ Department of Neurology, Ilsan Paik Hospital, Inje University, Goyang, Korea \\ 'Department of Neurology, Yonsei University College of Medicine, Seoul, Korea
}

\begin{abstract}
After recent randomized control trials (RCTs), non-vitamin K oral anticoagulants (NAOAs) are now widely being used in patients with atrial fibrillation (AF) worldwide. However, current guidelines for the use of NOACs in patients with AF are derived mostly using a Caucasian population and nonstroke patients. Relatively few Asian patients with AF and stroke are included in the recent RCTs. As a result, the optimal use of NOACs in this particular group of patients is remains to be settled. The optimal dose of NOACs and response to current dose of NOACs of Asian patients with AF and stroke may differ from those of westerners and patients without stroke. We reviewed available research on NOACs by searching PubMed and ClinicalTrials.gov published in English up to December 2015. In this review, the characteristics of Asian AF patients with prior stroke/transient ischemic attack, which might influence the efficacy and safety profiles of NOACs, are discussed. In addition, we summarize the risk factors for bleeding complications on NOACs, which are related or unrelated with the blood level of NOACs. Lastly, we provide recent data of reduced dose of NOACs from RCTs or large cohorts. The results reviewed herein call for clinical trials to test whether a reduced dose of NOACs is beneficial in Asian patients with AF and stroke. In the meantime, further researches are needed to establish the safety and efficacy of dose-adjusted NOACs considering both blood levels of NOACs and fragility of patients in Asian patients with AF and stroke.
\end{abstract}

Keywords Anticoagulation; Atrial fibrillation; Asians; Stroke; Ischemic stroke; Hemorrhage

\author{
Correspondence: Oh Young Bang \\ Department of Neurology, Samsung \\ Medical Center, Sungkyunkwan \\ University, 81 Irwon-ro, Gangnam-gu, \\ Seoul 06531, Korea \\ Tel: +82-2-3410-3599 \\ Fax: +82-2-3410-0052 \\ E-mail: ohyoung.bang@samsung.com
}

Received: January 23, 2016

Revised: March 1, 2016

Accepted: March 26, 2016

The authors have no financial conflicts of interest.

\section{Introduction}

Large randomized controlled trials (RCTs) consistently showed that non-vitamin $\mathrm{K}$ antagonist oral anticoagulants (NOACs) were at least as effective and safe as (regarding intracranial bleeding, much safer than) warfarin for stroke prevention in atrial fibrillation (AF), ${ }^{1-4}$ and regulatory authorities in many countries have approved NOACs. As a result, NOACs are now increasingly and widely being used worldwide. However, even with NOACs, the risk of major bleeding complication is not negligible; the annual major bleeding rates in the landmark RCTs were $2.71 \%$ and
$3.11 \%$ with low and high dose dabigatran, $3.13 \%$ with rivaroxaban, $2.8 \%$ with apixaban, and $1.61 \%$ and $2.75 \%$ with low and high dose edoxaban. ${ }^{1-4}$

Patients enrolled in RCTs are only partly representative of patients with AF in clinical practice. ${ }^{5}$ Furthermore, Asian AF patients with prior stroke or transient ischemic attack (TIA) constituted the minority of patients enrolled in the RCTs; $10.7 \%$ were Asians (from 6.5\% of the ROCKET-AF trial to $15.4 \%$ of the RE-LY trial), 20.3\% were patients with prior stroke or TIA (from 18.9\% in the ARISTOTLE trial to $52.4 \%$ of the ROCKET-AF trial), ${ }_{1}^{10-12}$ and thereby only $3.7 \%$ were Asian patients with prior stroke or TIA. ${ }^{13}$ 
The relative efficacy and safety of NOACs over warfarin appear to be greater in Asians than in non-Asians, ${ }^{13-15}$ but the bleeding rates with NOACs were higher in Asians than in non-Asians. Therefore, the optimal dose and response to the currently recommended dose of NOACs in Asian AF patients with prior stroke/TIA may differ from those in non-Asians and those without prior stroke/TIA.

In this review, we will discuss the characteristics of Asian AF patients with prior stroke/TIA, which might influence the efficacy and safety profiles of NOACs. In addition, we will summarize the risk factors for bleeding complications on NOACs, which are related or unrelated with the blood level of NOACs. Lastly, we will provide recent data of reduced dose of NOACs from RCTs or large cohorts.

\section{Search strategy and selection criteria}

We identified references for this review by searching PubMed and ClinicalTrials.gov published in English up to December 2015, with the search terms of AF, anticoagulation, Asians, stroke, cerebral infarction, bleeding, and hemorrhage. Additional relevant articles were identified from manual searches of relevant articles and reviews and were solicited from the authors. The final reference list was generated on the basis of originality and relevance to this topic. Because of space limitation, we were not able to include the results of small cohort studies and we described the results of RCTs only briefly.

\section{Summary of efficacy and safety findings from representative studies}

Table 1 summarizes the findings of efficacy and safety endpoints from representative RCTs, cohort studies, and meta-analyses.

In a meta-analysis of overall populations including Asians and non-Asians enrolled in 4 major RCTs, ${ }^{16}$ high-dose NOACs compared to warfarin significantly reduced stroke or systemic embolism by $19 \%$ (risk reduction $0.81,95 \% \mathrm{Cl} 0.73-0.91 ; P<0.001$ )

Table 1. Summary of risk of stroke/thromboembolism and major bleeding with and without dose reduction of non-vitamin K oral anticoagulants and warfarin

\begin{tabular}{|c|c|c|c|c|c|}
\hline \multirow[t]{2}{*}{ NOAC vs. warfarin } & \multirow[t]{2}{*}{ No. of patients } & \multirow{2}{*}{$\begin{array}{c}\text { Primary end point } \\
\qquad \mathrm{HR}(95 \% \mathrm{Cl}) \\
\text { Stroke or SE }\end{array}$} & \multicolumn{3}{|c|}{$\begin{array}{l}\text { Bleeding complications } \\
\text { HR }(95 \% \mathrm{Cl})\end{array}$} \\
\hline & & & Major bleeding & ICH & G-I bleeding \\
\hline \multicolumn{6}{|l|}{ RE-LY trial } \\
\hline Dabigatran $150 \mathrm{mg}$ & 6,076 & $0.66(0.53-0.82)$ & $0.93(0.81-1.07)$ & $0.40(0.27-0.60)$ & $1.50(1.19-1.89)$ \\
\hline Dabigatran $110 \mathrm{mg}$ & 6,015 & $0.91(0.74-1.11)^{*}$ & $0.80(0.55-0.83)^{*}$ & $0.31(0.20-0.47)$ & $1.10(0.86-1.41)^{*}$ \\
\hline \multicolumn{6}{|l|}{ U.S. Medicare data (propensity-matched) } \\
\hline Dabigatran $150 \mathrm{mg}$ & 56,576 & $0.70(0.57-0.85)$ & $N / A$ & $0.30(0.21-0.42)$ & $1.51(1.32-1.73)$ \\
\hline Dabigatran $75 \mathrm{mg}$ & 10,522 & $0.88(0.60-1.27)$ & N/A & $0.46(0.26-0.81)$ & $1.01(0.78-1.31)$ \\
\hline \multicolumn{6}{|c|}{ Taiwan national health insurance data (propensity-matched) } \\
\hline Dabigatran $150 \mathrm{mg}$ & 1,168 & $0.61(0.37-1.00)$ & N/A & $0.22(0.06-0.76)$ & $1.05(0.29-3.76)$ \\
\hline Dabigatran 110 mg & 8,772 & $0,62(0.52-0.75)^{*}$ & $\mathrm{~N} / \mathrm{A}$ & $0.47(0.34-0.65)$ & $0.99(0.64-1.52)$ \\
\hline \multicolumn{6}{|l|}{ ROCKET-AF trial } \\
\hline Rivaroxaban (20 mg in 79.4\%) & 7,081 & $0.79(0.66-0.96)$ & $1.04(0.90-1.20)$ & $0.67(0.47-0.93)$ & N/A \\
\hline Rivaroxaban (15 mg J-ROCKET) & 1,280 & $0.49(0.24-1.00)$ & $0.85(0.50-1.43)$ & $0.73(0.16-3.25)$ & $\mathrm{N} / \mathrm{A}$ \\
\hline ARISTOTLE trial (Apixaban) & 9,120 & $0.79(0.66-0.95)$ & $0.68(0.61-0.75)$ & $0.42(0.30-0.58)$ & $0.89(0.70-1.15)$ \\
\hline \multicolumn{6}{|l|}{ ENGAGE AF-TIMI 48 trial } \\
\hline Edoxaban $60 \mathrm{mg}$ & 5,251 & $0.87(0.73-1.04)$ & $0.80(0.71-0.91)$ & $0.47(0.34-0.63)$ & $1.23(1.02-1.50)$ \\
\hline Edoxaban $30 \mathrm{mg}$ & 1,784 & $1.13(0.96-1.34)^{*}$ & $0.47(0.41-0.55)^{*}$ & $0.30(0.21-0.43)^{*}$ & $0.67(0.53-0.83)^{*}$ \\
\hline \multicolumn{6}{|l|}{ Meta-analysis (Ruff et al. 2014) } \\
\hline High-dose NOACs & 29,287 & $0.81(0.73-0.91)$ & $0.86(0.73-1.00)$ & $0.48(0.39-0.59)$ & $1.25(1.01-1.25)$ \\
\hline Low-dose NOACs & 13,049 & $1.03(0.84-1.27)$ & $0.65(0.43-1.00)$ & $0.31(0.24-0.41)$ & $0.89(0.57-1.37)$ \\
\hline \multicolumn{6}{|l|}{ Meta-analysis (Wang et al. 2015) } \\
\hline Standard dose of NOACs in Asians & 3,035 & $0.65(0.52-0.83)$ & $0.57(0.44-0.74)$ & $0.33(0.22-0.50)$ & $0.79(0.48-1.32)$ \\
\hline Low dose of NOACs in Asians & 2,216 & $0.93(0.71-1.21)$ & $0.52(0.32-0.86)$ & $0.28(0.16-0.49)$ & $0.67(0.39-1.15)$ \\
\hline Standard dose of NOACs in non-Asians & 26,277 & $0.85(0.77-0.93)$ & $0.89(0.76-1.04)$ & $0.52(0.42-0.64)$ & $1.44(1.12-1.85)$ \\
\hline Low dose of NOACs in non-Asians & 11,473 & $1.07(0.93-1.24)$ & $0.64(0.38-1.09)$ & $0.32(0.24-0.44)$ & $0.87(0.56-1.35)$ \\
\hline
\end{tabular}

The meta-analysis for standard vs. low dose NOACs included data of dabigatran $150 \mathrm{vs.} 110 \mathrm{mg}$, edoxaban $60 \mathrm{vs.} 30 \mathrm{mg}$, and rivaroxaban $20 \mathrm{vs.} 15 \mathrm{mg}$. NOAC, non-vitamin K oral anticoagulant; HR, hazard ratio; Cl, confidence interval; SE, systemic embolism; ICH, Intracranial bleeding; G-I, Gastrointestinal; N/A, not assessed.

*Significant difference between two dose groups. 
and intracranial hemorrhage by $52 \%(0.48,0.39-0.59 ; P<$ $0.001)$, and there were no heterogeneity across the trials. However, the reduction of major bleeding with high-dose NOACs was non-significant $(0.86,0.73-1.00 ; P=0.06)$, and there was significant heterogeneity across the trials. Gastrointestinal bleeding was significantly increased with high-dose NOACs $(1.25,1.01$ $1.55 ; P=0.043)$, but significant heterogeneity was noted. In contrast, low-dose NOACs (110 mg dabigatran and 30/15 mg edoxaban) compared to warfarin had similar risk of stroke or systemic embolism $(1.03,0.84-1.27 ; P=0.74)$ and higher risk of ischemic stroke $(1.28,1.02-1.0 ; P=0.045)$, but had comparable risk of gastrointestinal bleeding $(0.89,0.57-1.37 ; P=0.58)$, non-significant reduction in major bleeding $(0.65,0.43-1.00 ; P=0.05)$, and substantial reduction in intracranial hemorrhage $(0.31,0.24-$ $0.41 ; P<0.001)$.

The relative safety and efficacy of NOACs compared to warfarin may be greater in Asians than in non-Asians, especially in stroke patients. ${ }^{14,15}$ In a recent meta-analysis, ${ }^{17}$ Asians had greater benefits of high-dose NOACs over warfarin than non-Asians for the endpoints of stroke or systemic embolism (odds ratio [95\% Cl], 0.65 [0.52-0.83] vs. 0.85 [0.77-0.93]; $P$ interaction $=0.045)$, major bleeding $(0.57[0.44-0.74]$ vs. 0.89 [0.76-
1.04], $P$ interaction $=0.004)$, and hemorrhagic stroke $(0.32$ [0.19$0.52]$ vs. $0.56[0.44-0.70], P$ interaction $=0.046$ ). While the risk of gastrointestinal bleeding was higher with high-dose NOACs than with warfarin in non-Asians (1.44 [1.12-1.85]), but not in Asians (0.79 [0.48-1.32]) $(P$ interaction $=0.041)$. In contrast, when comparing low-dose NOACs compared to warfarin, Asians and non-Asians did not differ in the results of efficacy and safety endpoints (Table 1).

In patients randomized to NOACs, Asians versus non-Asians had numerically lower rates of major bleeding except for those on edoxaban $60 \mathrm{mg}$ (Figure 1). However, the risks of intracranial hemorrhage, the most devastating complication, were higher in Asians than in non-Asians (the annual risk difference from 0\%/ year to $0.37 \%$ /year) and greater in patients with prior stroke/TIA than in those without (the annual risk difference from 0.2\%/year to $0.26 \% /$ year) (Figure 2). ${ }^{6-9}$ Therefore, these results suggest that intracranial hemorrhage rather than major bleeding (including gastrointestinal bleeding) is a major concern in selection and dosing of NOACs in Asians. Asian AF patients with prior stroke/ TIA who are likely to have fragile cerebrovascular bed might have greater risk of intracranial hemorrhage, but until now no analysis has been specifically conducted for these patients.
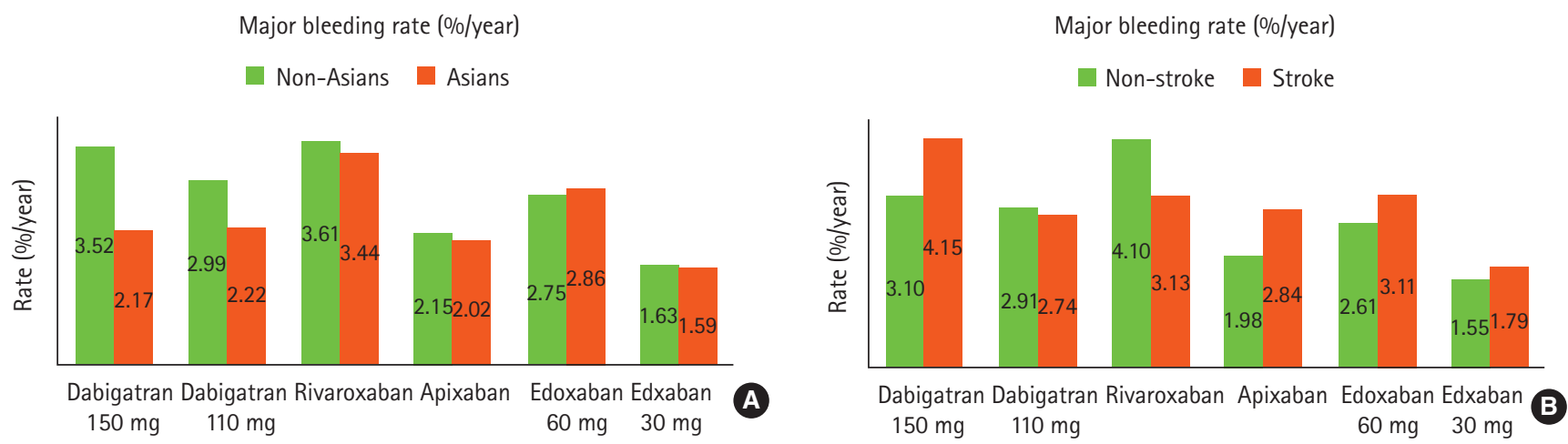

Figure 1. Annual rates of major bleeding with NOACs in (A) Asians vs. non-Asians and (B) patients with prior stroke/TIA vs. patients without from AF RCTs.
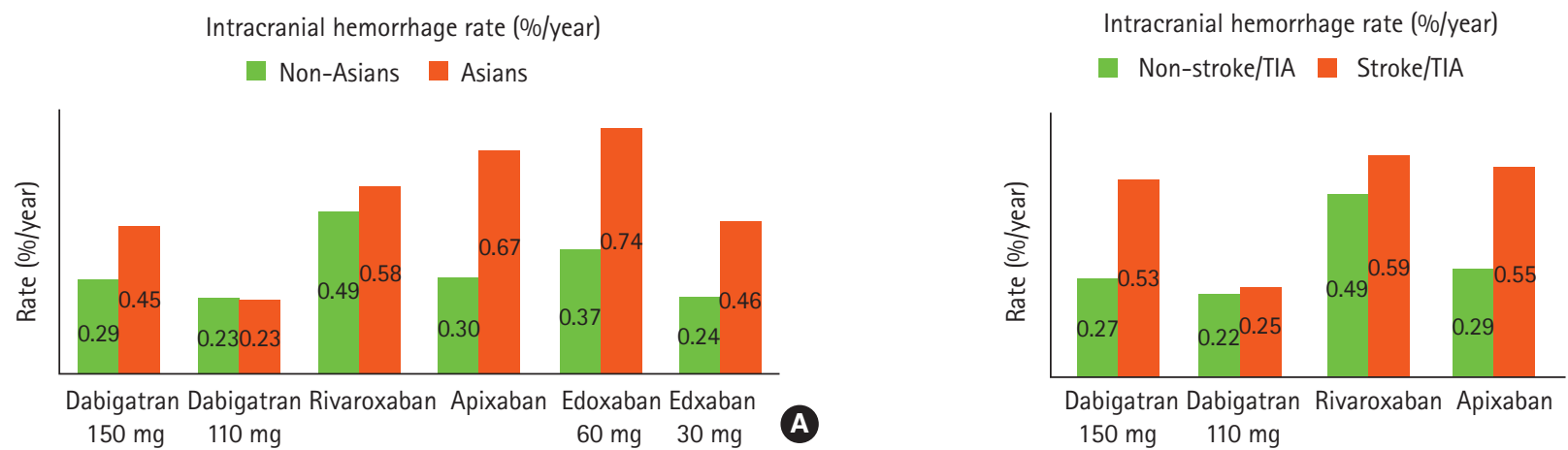

B

Figure 2. Annual rates of intracranial hemorrhage with NOACs in (A) Asians vs. non-Asians and (B) patients with prior stroke/TIA vs. patients without from AF RCTs. 


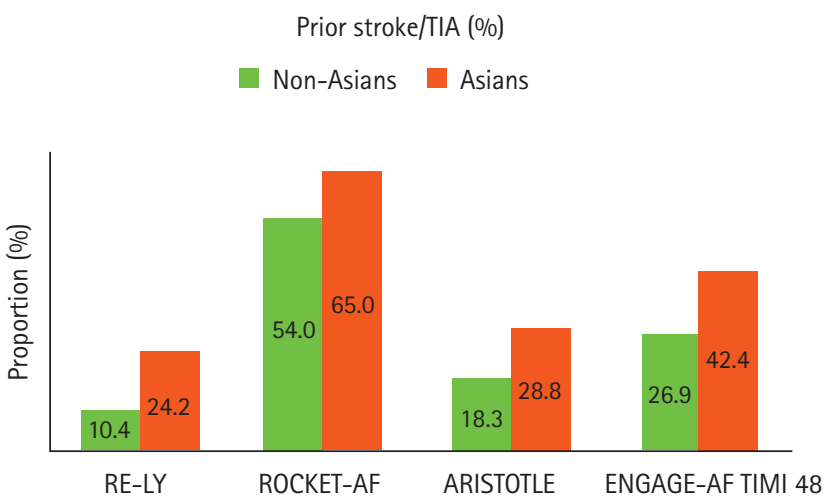

Figure 3. Patients with a prior stroke/TIA enrolled in NOACs RCTs.

\section{Characteristics of Asians patients with stroke and atrial fibrillation}

In multinational survey or cohort studies, Asian AF patients were more likely to have a history of prior stroke/TIA and lower body weights (or less obesity) than non-Asian AF patients. ${ }^{18-21}$ In a Japanese community-based registry of AF patients, the mean age was 74.2 years, the mean body weight was $58.5 \mathrm{~kg}, 21.8 \%$ had a history of stroke/TIA, and the mean creatinine clearance $(\mathrm{CrCl})$ was $63.4 \mathrm{~mL} / \mathrm{min}$ and $35.6 \%$ had moderate to severe renal impairment $(<30 \mathrm{~mL} / \mathrm{min}$ in $11.4 \%$ and $30-50 \mathrm{~mL} / \mathrm{min}$ in $24.2 \%) .{ }^{22}$ Similarly, among patients enrolled in 4 RCTs of NOACs, Asians had more history of stroke/TIA (despite the comparable CHADS2 score) and substantially lower body weights than nonAsians (Figures 3 and 4). ${ }^{6-9}$ The difference in body weight between Asians and non-Asians was $20 \mathrm{~kg}$ in RE-LY, $16 \mathrm{~kg}$ in ROCKET-AF, $17 \mathrm{~kg}$ in ARISTOTLE, and $20 \mathrm{~kg}$ in ENGAGE-AF TIMI 48. Body weight, along with serum creatinine level, age and sex determines $\mathrm{CrCl}$, which significantly influence NOACs metabolism. Of note, the proportions of patients with mild $(\mathrm{CrCl} 50-80$ $\mathrm{mL} / \mathrm{min})$ or moderate $(\mathrm{CrCl} 30-50 \mathrm{~mL} / \mathrm{min})$ renal impairment were higher in Asians than in non-Asians. ${ }^{6-9,23}$

Among Asian patients with $A F$, characteristics are likely to differ between patients with a history of stroke/TIA and those without, but no study has conducted formal comparison. In a Japanese multicenter registry of acute ischemic stroke patients with

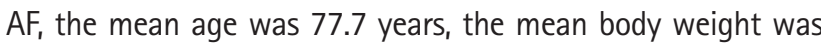
$56.3 \mathrm{~kg}$, and the mean $\mathrm{CrCl}$ was $56.6 \mathrm{~mL} / \mathrm{min}^{24}$ Despite the limitation of indirect comparison, Japanese AF patients with stroke/ TIA compared to Japanese overall AF patients appear to have higher age, lower body weight, and lower renal function. ${ }^{22,24}$ In a single center study of Korea, AF patients with stroke/TIA had a mean age of 73.9 years and $\mathrm{CrCl}$ of $56.8 \mathrm{~mL} / \mathrm{min}^{5}$ which were similar to those of Japanese AF patients with stroke/TIA. In this study, when the enrollment criteria of four RCTs were applied to

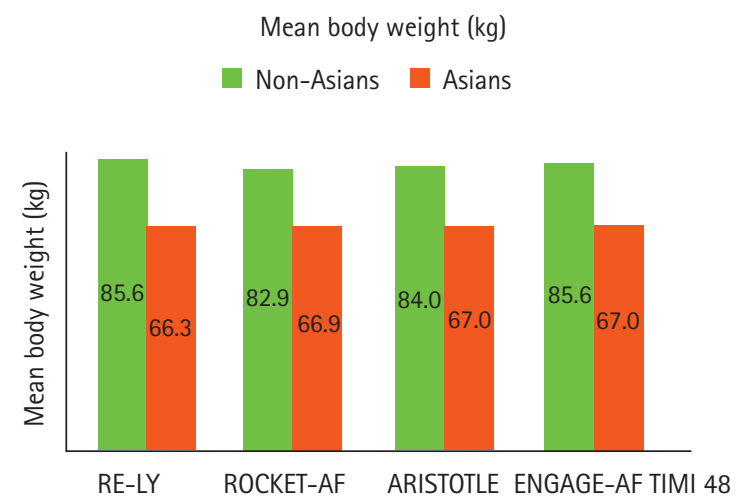

Figure 4. Body weight of patients enrolled in NOACs RCTs.

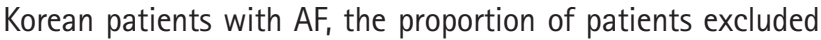
from trials were expected to be higher in patients with stroke/ TIA (27.2\%-42.6\%, depending on individual trial's criteria) vs. those without stroke/TIA (18.5\%-27.2\%). The main reasons for ineligibility for RCTs in patients with stroke/TIA were high of bleeding risk (15.2\%-20.8\%) and low $\mathrm{CrCl}$ (5.6\%-9.2\%).

Compared to non-Asians, Asians are at higher risk of warfarinrelated intracranial bleeding, ${ }_{1}^{25}$ and the prevalence of intracranial macro- and microangiopathy also appears to be high. ${ }^{26-28}$ For example, cerebral microbleeds, which are high prevalent in Asians and stroke patients, increase the risk of intracranial bleeding with warfarin use. ${ }^{29-31}$ Individualized antithrombotic therapy, e.g., use of NOACs, was proposed depending on the presence of and increase in the number of cerebral microbleeds on brain MRI. ${ }^{32,33}$

Previous ischemic stroke or TIA is the most powerful independent risk factor for stroke in patients with $\mathrm{AF}^{34}$ The early period after acute ischemic stroke or TIA is particularly at high risk of recurrent stroke, but also at high risk of intracranial bleeding. Furthermore, a large population-based study showed an increased risk of stroke during the early period of warfarin initiation, ${ }^{35}$ possibly due to prothrombotic activity of warfarin at treatment initiation. ${ }^{36}$ For anticoagulation in acute cerebral ischemia, NOACs might be better than warfarin. However, patients with recent stroke/TIA were largely excluded in the landmark NOAC AF trials: the cut-off time point for eligibility from stroke onset to randomization was 30 days in ENGAGE-AF, 14 days in RE-LY and ROCKET-AF, and 7 days in ARISTOTLE. As a result, data about the use of NOACs in patients with recent stroke are lacking. Currently, a randomized trial is ongoing to examine the safety and efficacy of early initiation (within 5 days from stroke onset) of rivaroxaban in patients with AF and acute ischemic stroke (TripleAXEL, NCT02042534). ${ }^{37}$

In patients with $A F$, stroke is not always caused by cardiogenic embolism (AF-unrelated mechanism). Since macro- and microangiopathies are more prevalent in Asians than in non-Asians, 
AF-unrelated stroke/TIA is more likely to occur in Asian AF patients than in non-Asian AF patients. In a Korean study, among AF patients with recent ischemic stroke, $17.2 \%$ of ischemic strokes were classified as AF-unrelated strokes. ${ }^{38}$ Moreover, compared to patients with AF-related stroke, patients with AF-unrelated stroke experienced more recurrent strokes even with adequate anticoagulation, and $87.5 \%$ of their recurrent strokes were AF-unrelated strokes. ${ }^{38}$ Therefore, it would be of clinical relevance to differentiate stroke mechanism in AF patients. A recent multidetector cardiac computed tomography study showed that morphometric and volumetric changes of left atrial appendage was not prominent in AF-unrelated stroke. ${ }^{39}$ For patients with AF-related stroke, antiplatelet drugs should be discontinued at the time of NOACs initiation unless there is a strong indication other than secondary stroke prevention for their continuous use. However, antiplatelet therapy might be often needed in patients with AF-unrelated stroke, particularly during the early period after acute cerebral ischemia.

Annual risk of stroke in individuals with $\mathrm{AF}$ is a continuum and increases with risk factors. The $\mathrm{CHADS}_{2}$ and $\mathrm{CHA}_{2} \mathrm{DS}_{2}$-VASc schemes are most widely used model for stratification of AF-related stroke risks, but a high score would also indicate a higher atherosclerotic burden. Previous studies showed that high $\mathrm{CHADS}_{2}$ and $\mathrm{CHA}_{2} \mathrm{DS}_{2}$-VASc scores were associated with subsequent stroke, cardiovascular events, and death in stroke patients without $\mathrm{AF}^{40}$ and in acute coronary syndrome patients. ${ }^{41}$ Thereby, AF patients with high $\mathrm{CHADS}_{2}$ or $\mathrm{CHA}_{2} \mathrm{DS}_{2}-\mathrm{VASc}$ scores might have a higher risk of AF-unrelated stroke (macro- or microangiopathy). ${ }^{42,43}$ A large Chinese cohort of AF patients showed that although net benefit favor warfarin over antiplatelet for patients at high risk of stroke, the benefit of warfarin decreased with the increase in the CHA2DS2-VASc scores. ${ }^{44}$

Therefore, concomitant use of antiplatelet agents are often needed in Asian stroke patients with AF. In the RE-LY trial, Asians had a higher rate of previous stroke (24.2\% vs. $10.4 \%)$ and use of antiplatelet agents ( $47.1 \%$ vs. $38.1 \%$ ) than non-Asians. ${ }^{6}$ Concomitant use of antiplatelet agents and anticoagulants increase the bleeding risks. A meta-analysis of patients receiving antiplatelet therapy after an acute coronary syndrome showed that the use of NOACs is associated with a dramatic increase ( 3 times) in major bleeding events. ${ }^{45}$

\section{Dose of NOACs: serum level vs. fragility of patients}

\section{Renal function, a major determinant of serum NOAC levels}

Based on the study design and results of subgroup analysis of
RCTs of NOACs, current guidelines of AF management recommended dose reduction according to the serum level of NOACs. ${ }^{46}$ Because NOACs are predominantly or partially excreted by the kidneys, only patients with $\mathrm{CrCl} \geq 30 \mathrm{~mL} / \mathrm{min}$ (dabigatran and rivaroxaban) or $\geq 25 \mathrm{~mL} / \mathrm{min}$ (apixaban) were included in the trials. The studies with apixaban and rivaroxaban used reduced doses for patients with $\mathrm{CrCl}<50 \mathrm{~mL} / \mathrm{min}$. Age ( $>80$ years) for dabigatran and apixaban, body weight ( $\leq 60 \mathrm{~kg}$ ) for apixaban and edoxaban, and the use of P-glycoprotein antagonist (e.g., dronedarone) were also considered in selection of NOAC dose. Both age and body weight are parameters used to estimate $\mathrm{CrCl}$ by Cockcroft-Gault equation, and absorption of NOACs is dependent on the intestinal P-glycoprotein system.

However, the benefits of the NOACs seem to be larger in chronic kidney disease stages. ${ }^{47}$ In the ARISTOTLE trial, relative reduction in major bleeding was greater in patients with renal dysfunction. ${ }^{48}$ For Asian patients with stroke, data about bleeding risk depending on the renal function is limited. In Asian patients in the RE-LY trial, bleeding rate was increased with renal impairment, but relative benefits of dabigatran over warfarin were preserved regardless of renal function. ${ }^{49}$

\section{Comorbid conditions of patients, major determinants of bleeding complications}

Safe use of NOACs needs understanding of when to reduce the dose of NOACs. In this context, it is possible that fragility of patients (clinical and radiological features) may be as important as serum NOAC levels (related to renal function) in the development of major bleeding during the use of NOACs.

Post-hoc analysis of RCTs showed determinants of major bleeding in NOAC users. Elderly, prior stroke, prior gastrointestinal bleeding/anemia, aspirin use, renal dysfunction, and multiple cerebral microbleeds were commonly associated with major bleeding. ${ }^{50-52}$ Patients with AF and stroke often have comorbid conditions and take multiple medications. In ROCKET AF, twothirds of enrolled patients were on $\geq 5$ medications, and the bleeding risk increased as the medication numbers increased. ${ }^{53}$ In RE-LY, concomitant antiplatelet drugs increased the risk of major bleeding, and the major bleeding risk was higher with dual antiplatelet therapy than with single antiplatelet therapy. Of patients who had concomitant antiplatelet use, the major bleeding risk was lowest in low dabigatran user (dabigatran $110 \mathrm{mg}, \mathrm{BID}$ ). ${ }^{54}$

It is interesting that many of the factors are relatively unrelated to the degree of renal impairment, suggesting the importance of consideration of 'non-renal' factors as well as renal factor when considering the dose of NOACs (Figure 5). 


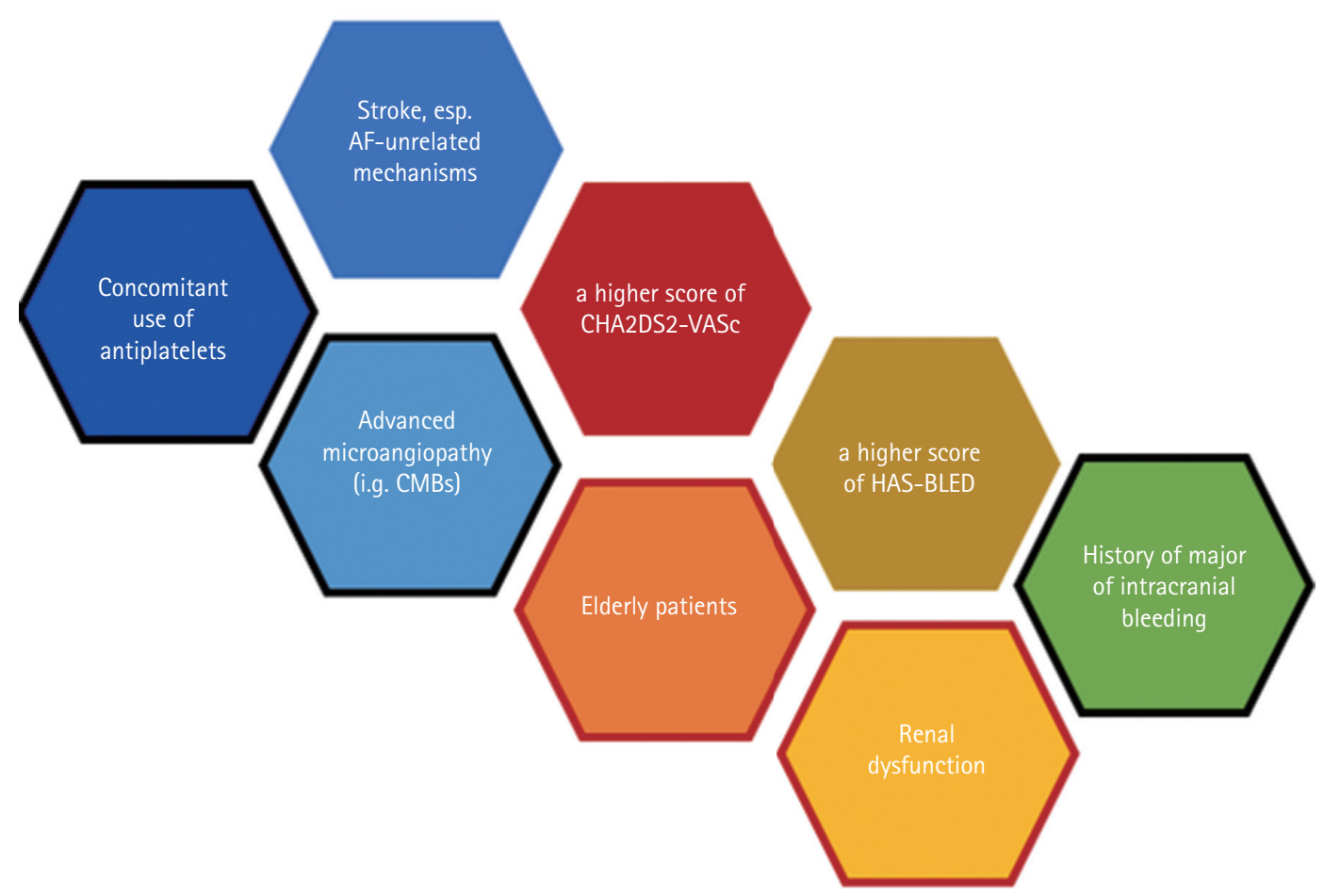

Figure 5. Factors associated with major or intracranial bleeding in RCTs of NOAC trials and their relationships. Black outlines represent fragility in Asian patients with stroke, whereas red outlines represent factors that determined serum levels of NOACs. CMBs, cerebral microbleeds.

\section{Results of low doses of warfarin and non-vitamin K oral anticoagulants}

\section{Need for a low level of anticoagulation in frail patients}

Anticoagulation therapy is needed even in frail patients with AF who have an increased risk of bleeding. A Japanese community-based survey (Fushimi AF registry) showed that features suggesting fragility of patients (i.e., advanced age, underweight, previous stroke, heart failure, chronic kidney disease, and anemia) were independently associated with risk of stroke and death in non-anticoagulated patients..$^{55}$ In post-hoc analysis of ROCK$E T-A F$, renal dysfunction was a strong, independent predictor of stroke and systemic embolism and a risk estimation scheme incorporating renal dysfunction ( $\mathrm{R}_{2} \mathrm{CHADS}_{2}$ score) better predicted the risk of stroke or systemic embolism in ROCKET-AF and ATRIA (AnTicoagulation and Risk factors In Atrial fibrillation) populations. $^{56}$

A Japanese study (J-RHYTHM registry) showed that warfarin could have beneficial effects even in very old patients with $A F$ if INR is kept between 1.6 and 2.59, suggesting importance of a lower level of anticoagulation in frail patients. ${ }^{57}$

The benefits of NOACs compared with warfarin in the elderly individuals with multiple comorbid conditions were consistently observed among the trials. ${ }^{58}$ NOACs appear promising and help overcome some if not all of the limitations of warfarin in early frail patients. ${ }^{58}$ In fact, the clinical benefits of NOACs may be greater in patients with high bleeding risk. ${ }^{59}$ Proposals for decision-making in NOACs use for these frail/high bleeding risk patients were recently suggested. ${ }^{58-60}$ However, in the real world, NOACs were underutilized in these patients (e.g., elderly). ${ }^{61,62}$ Our results of Korean patients with $\mathrm{AF}$ and stroke showed that there was a negative correlation between $\mathrm{CrCl}$ and ATRIA score (a scheme for bleeding-risks), and patients who had features favoring NOAC use (i.e., a high ATRIA score) were more likely to have contraindications for NOACs use (i.e., a low $\mathrm{CrCl})^{5}$

\section{Evidences from randomized controlled trial}

RCTs and large cohort studies have evaluated the safety and efficacy of different doses of NOACs in patients with AF. In particular, the RE-LY and ENGAGE AF-TIMI 48 trials formally tested two different doses of NOACs (Table 1).

In the J-ROCKET-AF study, $15 \mathrm{mg}$ rivaroxaban instead of 20 $\mathrm{mg}$ was used, ${ }_{1}^{63}$ because $15 \mathrm{mg}$ rivaroxaban dose in Japanese patients yield exposures comparable to $20 \mathrm{mg}$ dose in white patients. ${ }^{64}$ The trial results demonstrated noninferiority of $15 \mathrm{mg}$ rivaroxaban and less intracranial bleeding over warfarin, which supports the use of a reduced dose of rivaroxaban $(15 \mathrm{mg})$ for evaluation in Japanese patients with $A F^{63}$

The ENGAGE AF-TIMI 48 trial evaluating the efficacy of edoxa- 
ban vs. warfarin in patients with AF provided the evidence of supporting the use of a lower dose of NOAC in fragile patient population. ${ }^{4,65}$ In this study, low-dose ( $30 \mathrm{mg}$ vs. $60 \mathrm{mg}$ ) edoxaban was associated with a $41 \%$ increase in ischemic stroke, which is counterbalanced by a 53\% reduction in hemorrhagic stroke, suggesting an Importance of the low dose of NOACs in fragile patient populations that present with greater risk of hemorrhagic stroke. Despite the lower anti-factor Xa activity, dose reduction preserved the efficacy of edoxaban compared with warfarin, suggesting that the therapeutic window for edoxaban is narrower for major bleeding than thromboembolism. ${ }^{65}$ One recent Japanese study showed that bleeding rate, plasma edoxaban concentration and biomarkers of blood coagulation and fibrinolysis were similar between patients with severe renal impairment receiving low dose edoxaban $(15 \mathrm{mg})$ and those with normal renal function receiving a higher dose edoxaban $(30$ or $60 \mathrm{mg}){ }^{66}$

Similar results were also observed in the RE-LY trial that showed dose reduction of dabigatran significantly increased in stroke or systemic embolism but a significant reduction in major bleeding.' However, further studies are needed because, in the recent study of propensity score matched elderly patients enrolled in Medicare, neither stroke/systemic embolism nor intracranial/major gastrointestinal bleeding were significantly different between patients received dabigatran $150 \mathrm{mg}$ vs. $75 \mathrm{mg}$ twice daily. ${ }^{67}$ Very recently, the Taiwan national health insurance research database showed that $88 \%$ patients took $110 \mathrm{mg}$ dabigatran and the magnitude of effect for outcomes of $110 \mathrm{mg}$ was comparable with those of $150 \mathrm{mg}$ dose in real-world practice. ${ }^{68}$

A recent meta-analysis of RCTs of NOACs comparing efficacy and safety of NOACs between patients enrolled in Asian and non-Asian countries showed that standard-dose NOACs are preferred over warfarin in Asians patients, whereas low-dose NOACs are effective and safe alternative to warfarin. ${ }^{17}$

\section{Perspectives and conclusions}

Current guidelines for the use of NOACs in patients with AF are largely derived from Caucasian and non-stroke patients. Asian patients with AF and stroke might be 'specific' AF population due to 'specific' profiles of higher risks of both thromboembolic and bleeding events, and both patients and physicians are often reluctant to use NOACs as well as warfarin due to fear of bleeding complications. However, no specific guidelines exist regarding the dose of NOACs in these patients. ${ }^{69,70}$ Measurement of serum levels of NOACs and anti-lla or anti-Xa activity may improve safety during NOAC therapy, but not available in clinical practice yet. Several clinical and radiological features related bleeding complications may provide additional information to current guideline (dose reduction according to renal function to achieve optimal serum NOAC levels) in the selection of dose of NOACs.

Available data reviewed herein suggest that anticoagulation with NOACs could be promising and help overcome limitations of warfarin, especially in elderly frail patients, such as Asian patients with AF and stroke. Although the dose of NOACs should be used under the current guidelines for NOAC use in patients with $A F$, emerging evidences suggested that a reduced dose of NOACs could be helpful in these patients. To date, no studies have been conducted to test whether a reduced dose of NOACs is beneficial in Asian patients with AF and stroke. Because headto-head comparisons of NOACs regarding their efficacy in preventing stroke in these particular patients are lacking, a recommendation for a preferred use of any of the NOACs can not be made. In the meantime, further researches are needed to establish the safety and efficacy of dose-adjusted NOACs considering both blood level of NOACs and fragility of patients in Asian patients with $\mathrm{AF}$ and stroke.

\section{References}

1. Connolly SJ, Ezekowitz MD, Yusuf S, Eikelboom J, Oldgren J, Parekh $A$, et al. Dabigatran versus warfarin in patients with atrial fibrillation. N Engl J Med 2009;361:1139-1151.

2. Patel MR, Mahaffey KW, Garg J, Pan G, Singer DE, Hacke W, et al. Rivaroxaban versus warfarin in nonvalvular atrial fibrillation. N Engl J Med 2011;365:883-891.

3. Granger $\mathrm{CB}$, Alexander JH, McMurray JJ, Lopes RD, Hylek EM, Hanna $M$, et al. Apixaban versus warfarin in patients with atrial fibrillation. N Engl J Med 2011;365:981-992.

4. Giugliano RP, Ruff CT, Braunwald E, Murphy SA, Wiviott SD, Halperin $\mathrm{JL}$, et al. Edoxaban versus warfarin in patients with atrial fibrillation. N Engl J Med 2013;369:2093-2104.

5. Yoon $\mathrm{CH}$, Park YK, Kim SJ, Lee MJ, Ryoo S, Kim GM, et al. Eligibility and preference of new oral anticoagulants in patients with atrial fibrillation: comparison between patients with versus without stroke. Stroke 2014;45:2983-2988.

6. Hori M, Connolly SJ, Zhu J, Liu LS, Lau CP, Pais P, et al. Dabigatran versus warfarin: effects on ischemic and hemorrhagic strokes and bleeding in Asians and non-Asians with atrial fibrillation. Stroke 2013;44:1891-1896.

7. Wong KS, Hu DY, Oomman A, Tan RS, Patel MR, Singer DE, et al. Rivaroxaban for stroke prevention in East Asian patients from the ROCKET AF trial. Stroke 2014;45:1739-1747.

8. Goto S, Zhu J, Liu L, Oh BH, Wojdyla DM, Aylward P, et al. Efficacy and safety of apixaban compared with warfarin for stroke prevention in patients with atrial fibrillation from East Asia: a 
subanalysis of the apixaban for reduction in stroke and other thromboembolic events in atrial fibrillation (ARISTOTLE) trial. Am Heart J 2014;168:303-309.

9. Shimada YJ, Yamashita T, Koretsune Y, Kimura T, Abe K, Sasaki $S$, et al. Effects of regional differences in Asia on efficacy and safety of edoxaban compared with warfarin- insights from the ENGAGE AF-TIMI 48 trial. Circ J 2015;79:2560-2567.

10. Diener HC, Connolly SJ, Ezekowitz MD, Wallentin L, Reilly PA, Yang $S$, et al. Dabigatran compared with warfarin in patients with atrial fibrillation and previous transient ischaemic attack or stroke: a subgroup analysis of the RE-LY trial. Lancet Neurol 2010;9:1157-1163.

11. Hankey GJ, Patel MR, Stevens SR, Becker RC, Breithardt G, Carolei $A$, et al. Rivaroxaban compared with warfarin in patients with atrial fibrillation and previous stroke or transient ischaemic attack: a subgroup analysis of ROCKET AF. Lancet Neurol 2012;11:315-322.

12. Easton JD, Lopes RD, Bahit MC, Wojdyla DM, Granger CB, Wallentin $L$, et al. Apixaban compared with warfarin in patients with atrial fibrillation and previous stroke or transient ischaemic attack: a subgroup analysis of the ARISTOTLE trial. Lancet Neurol 2012;11:503-511.

13. Lip GY, Wang KL, Chiang CE. Non-vitamin k antagonist oral anticoagulants (NOACs) for stroke prevention in Asian patients with atrial fibrillation: time for a reappraisal. Int J Cardio/ 2015; 180:246-254.

14. Bang OY, Hong KS, Heo JH, Koo J, Kwon SU, Yu KH, et al. New oral anticoagulants may be particularly useful for Asian stroke patients. J Stroke 2014;16:73-80.

15. Chiang CE, Wang KL, Lip GY. Stroke prevention in atrial fibrillation: an Asian perspective. Thromb Haemost 2014;111:789797.

16. Ruff CT, Giugliano RP, Braunwald E, Hoffman EB, Deenadayalu $\mathrm{N}$, Ezekowitz MD, et al. Comparison of the efficacy and safety of new oral anticoagulants with warfarin in patients with atrial fibrillation: a meta-analysis of randomised trials. Lancet 2014;383:955-962.

17. Wang KL, Lip GY, Lin SJ, Chiang CE. Non-vitamin k antagonist oral anticoagulants for stroke prevention in Asian patients with nonvalvular atrial fibrillation: meta-analysis. Stroke 2015; 46:2555-2561.

18. Le Heuzey JY, Breithardt G, Camm J, Crijns H, Dorian P, Kowey $P R$, et al. The RecordAF study: design, baseline data, and profile of patients according to chosen treatment strategy for atrial fibrillation. Am J Cardiol 2010;105:687-693.

19. Amerena J, Chen SA, Sriratanasathavorn C, Cho JG, Huang D, Omar $\mathrm{R}_{1}$ et al. Insights into management of atrial fibrillation in Asia Pacific gained from baseline data from registry on cardiac rhythm disorders (RecordAF-Asia Pacific [AP]) registry. Am J Cardiol 2012;109:378-382.

20. Gamra H, Murin J, Chiang CE, Naditch-Brule L, Brette S, Steg PG. Use of antithrombotics in atrial fibrillation in Africa, Europe, Asia and South America: Insights from the international RealiseAF survey. Arch Cardiovasc Dis 2014;107:77-87.

21. Oldgren J, Healey JS, Ezekowitz M, Commerford P, Avezum A, Pais $P$, et al. Variations in cause and management of atrial fibrillation in a prospective registry of 15,400 emergency department patients in 46 countries: The RE-LY atrial fibrillation registry. Circulation 2014;129:1568-1576.

22. Akao $M$, Chun $Y H$, Wada $H$, Esato $M$, Hashimoto $T$, Abe $M$, et al. Current status of clinical background of patients with atrial fibrillation in a community-based survey: the Fushimi AF registry. J Cardiol 2013;61:260-266.

23. Miyamoto K, Nakasuka K, Kusano K. Effect of renal function on anticoagulation therapy in Asian patients. Circ J 2015;79:20982099.

24. Toyoda K, Arihiro S, Todo K, Yamagami H, Kimura K, Furui E, et al. Trends in oral anticoagulant choice for acute stroke patients with nonvalvular atrial fibrillation in Japan: the SAMURAI-NVAF study. Int J Stroke 2015;10:836-842.

25. Shen AY, Yao JF, Brar SS, Jorgensen MB, Chen W. Racial/ethnic differences in the risk of intracranial hemorrhage among patients with atrial fibrillation. J Am Coll Cardiol 2007;50:309315.

26. Koennecke HC. Cerebral microbleeds on MRI: Prevalence, associations, and potential clinical implications. Neurology 2006; 66:165-171.

27. Bang OY, Saver JL, Liebeskind DS, Pineda S, Yun SW, Ovbiagele B. Impact of metabolic syndrome on distribution of cervicocephalic atherosclerosis: data from a diverse race-ethnic group. J Neurol Sci 2009;284:40-45.

28. Tsai CF, Thomas B, Sudlow CL. Epidemiology of stroke and its subtypes in Chinese vs white populations: a systematic review. Neurology 2013;81:264-272.

29. Lovelock CE, Cordonnier C, Naka H, Al-Shahi Salman R, Sudlow $\mathrm{CL}$, Edinburgh Stroke Study Group, et al. Antithrombotic drug use, cerebral microbleeds, and intracerebral hemorrhage: a systematic review of published and unpublished studies. Stroke 2010;41:1222-1228.

30. Song TJ, Kim J, Song D, Nam HS, Kim YD, Lee HS, et al. Association of cerebral microbleeds with mortality in stroke patients having atrial fibrillation. Neurology 2014;83:1308-1315.

31. Akoudad S, Darweesh SK, Leening MJ, Koudstaal PJ, Hofman A, van der Lugt $A$, et al. Use of coumarin anticoagulants and cerebral microbleeds in the general population. Stroke 2014;45: 3436-3439. 
32. Fisher M. MRI screening for chronic anticoagulation in atrial fibrillation. Front Neurol 2013;4:137.

33. Wang Z, Soo YO, Mok VC. Cerebral microbleeds: is antithrombotic therapy safe to administer? Stroke 2014;45:2811-2817.

34. The Stroke Risk in Atrial Fibrillation Working Group. Independent predictors of stroke in patients with atrial fibrillation: a systematic review. Neurology 2007;69:546-554.

35. Azoulay L, Dell'Aniello S, Simon TA, Renoux C, Suissa S. Initiation of warfarin in patients with atrial fibrillation: early effects on ischaemic strokes. Eur Heart J 2014;35:1881-1887.

36. Kim Y, Bang OY. Paradoxical procoagulant effect of early doses of warfarin: possible role of non-vitamin $\mathrm{K}$ oral anticoagulant in patients with atrial fibrillation-related stroke. J Stroke 2015; 17:216-218.

37. Hong KS, Choi YJ, Kwon SU. Rationale and design of TRIPLE AXEL: trial for early anticoagulation in acute ischemic stroke patients with nonvalvular atrial fibrillation. Int J Stroke 2015; 10:128-133.

38. Kim SJ, Ryoo S, Kwon S, Park YK, Kim JP, Lee GY, et al. Is atrial fibrillation always a culprit of stroke in patients with atrial fibrillation plus stroke? Cerebrovasc Dis 2013;36:373-382.

39. Jeong WK, Choi JH, Son JP, Lee S, Lee MJ, Choe YH, et al. Volume and morphology of left atrial appendage as determinants of stroke subtype in patients with atrial fibrillation. Heart Rhythm 2016;13:820-827.

40. Ntaios G, Lip GY, Makaritsis K, Papavasileiou V, Vemmou A, Ko-

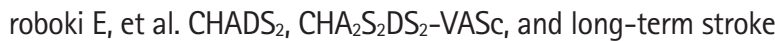
outcome in patients without atrial fibrillation. Neurology 2013; 80:1009-1017.

41. Chua SK, Lo HM, Chiu CZ, Shyu KG. Use of $\mathrm{CHADS}_{2}$ and $\mathrm{CHA}_{2} \mathrm{~S}_{2}$ $D_{2}$-VASc scores to predict subsequent myocardial infarction, stroke, and death in patients with acute coronary syndrome: data from Taiwan acute coronary syndrome full spectrum registry. PLoS One 2014;9:e111167.

42. Kim YD, Cha MJ, Kim J, Lee DH, Lee HS, Nam CM, et al. Increases in cerebral atherosclerosis according to $\mathrm{CHADS}_{2}$ scores in patients with stroke with nonvalvular atrial fibrillation. Stroke 2011;42:930-934.

43. Cha MJ, Kim YD, Nam HS, Kim J, Lee DH, Heo JH. Stroke mechanism in patients with non-valvular atrial fibrillation according to the $\mathrm{CHADS}_{2}$ and $\mathrm{CHA}_{2} \mathrm{~S}_{2} \mathrm{DS}_{2}$-VASc scores. Eur J Neurol 2012;19:473-479.

44. Siu CW, Lip GY, Lam KF, Tse HF. Risk of stroke and intracranial hemorrhage in 9727 Chinese with atrial fibrillation in Hong Kong. Heart rhythm 2014;11:1401-1408.

45. Komocsi A, Vorobcsuk A, Kehl D, Aradi D. Use of new-generation oral anticoagulant agents in patients receiving antiplatelet therapy after an acute coronary syndrome: systematic re- view and meta-analysis of randomized controlled trials. Arch Intern Med 2012;172:1537-1545.

46. Kaatz $\mathrm{S}$, Mahan CE. Stroke prevention in patients with atrial fibrillation and renal dysfunction. Stroke 2014:45:2497-2505.

47. Reinecke $H$, Engelbertz $C$, Schabitz WR. Preventing stroke in patients with chronic kidney disease and atrial fibrillation: benefit and risks of old and new oral anticoagulants. Stroke 2013;44:2935-2941.

48. Hohnloser SH, Hijazi Z, Thomas L, Alexander JH, Amerena J, Hanna $M$, et al. Efficacy of apixaban when compared with warfarin in relation to renal function in patients with atrial fibrillation: insights from the ARISTOTLE trial. Eur Heart J 2012; 33:2821-2830.

49. Hori M, Fukaya $T$, Kleine E, Reilly PA, Ezekowitz MD, Connolly SJ, et al. Efficacy and safety of dabigatran etexilate vs. Warfarin in Asian RE-LY patients according to baseline renal function or CHADS2 score. Circ J 2015;79:2138-2147.

50. Hart RG, Diener HC, Yang S, Connolly SJ, Wallentin L, Reilly PA, et al. Intracranial hemorrhage in atrial fibrillation patients during anticoagulation with warfarin or dabigatran: the RE-LY trial. Stroke 2012;43:1511-1517.

51. Goodman SG, Wojdyla DM, Piccini JP, White HD, Paolini JF, Nessel CC, et al. Factors associated with major bleeding events: insights from the ROCKET AF trial (rivaroxaban oncedaily oral direct factor $\mathrm{Xa}$ inhibition compared with vitamin $\mathrm{K}$ antagonism for prevention of stroke and embolism trial in atrial fibrillation). J Am Coll Cardiol 2014;63:891-900.

52. Hylek EM, Held C, Alexander JH, Lopes RD, De Caterina R, Wojdyla DM, et al. Major bleeding in patients with atrial fibrillation receiving apixaban or warfarin: the ARISTOTLE trial (apixaban for reduction in stroke and other thromboembolic events in atrial fibrillation): predictors, characteristics, and clinical outcomes. J Am Coll Cardiol 2014;63:2141-2147.

53. Piccini JP, Hellkamp AS, Washam JB, Becker RC, Breithardt G, Berkowitz SD, et al. Polypharmacy and the efficacy and safety of rivaroxaban versus warfarin in the prevention of stroke in patients with nonvalvular atrial fibrillation. Circulation 2016; 133:352-360.

54. Dans AL, Connolly SJ, Wallentin L, Yang S, Nakamya J, Brueckmann $M$, et al. Concomitant use of antiplatelet therapy with dabigatran or warfarin in the randomized evaluation of longterm anticoagulation therapy (RE-LY) trial. Circulation 2013; 127:634-640.

55. Hamatani $Y$, Yamashita $Y$, Esato $M$, Chun $Y H$, Tsuji $H$, Wada $H$, et al. Predictors for stroke and death in non-anticoagulated Asian patients with atrial fibrillation: The Fushimi AF registry. PLoS One 2015;10:e0142394.

56. Piccini JP, Stevens SR, Chang $Y$, Singer DE, Lokhnygina $Y$, Go 
AS, et al. Renal dysfunction as a predictor of stroke and systemic embolism in patients with nonvalvular atrial fibrillation: validation of the $\mathrm{R}_{2} \mathrm{CHADS}_{2}$ index in the ROCKET AF (rivaroxaban once-daily, oral, direct factor $\mathrm{Xa}$ inhibition compared with vitamin $\mathrm{K}$ antagonism for prevention of stroke and embolism trial in atrial fibrillation) and ATRIA (anticoagulation and risk factors in atrial fibrillation) study cohorts. Circulation 2013; 127:224-232.

57. Kodani $E_{1}$ Atarashi $H$, Inoue H, Okumura K, Yamashita T, Origasa $\mathrm{H}$. Use of warfarin in elderly patients with non-valvular atrial fibrillation- subanalysis of the J-rhythm registry. Circ J 2015;79:2345-2352.

58. Turagam MK, Velagapudi P, Flaker GC. Stroke prevention in the elderly atrial fibrillation patient with comorbid conditions: focus on non-vitamin $\mathrm{K}$ antagonist oral anticoagulants. Clin Interv Aging 2015;10:1431-1444.

59. Potpara TS, Lip GY. Oral anticoagulant therapy in atrial fibrillation patients at high stroke and bleeding risk. Prog Cardiovasc Dis 2015;58:177-194.

60. Verheugt FW, Granger CB. Oral anticoagulants for stroke prevention in atrial fibrillation: Current status, special situations, and unmet needs. Lancet 2015;386:303-310.

61. Lip GY, Laroche C, Dan GA, Santini M, Kalarus Z, Rasmussen LH, et al. 'Real-world' antithrombotic treatment in atrial fibrillation: the EORP-AF pilot survey. Am J Med 2014;127:519-529. e1.

62. Lopatowska P, Tomaszuk-Kazberuk A, Mlodawska E, Bachorzewska-Gajewska $\mathrm{H}_{1}$ Malyszko J, Dobrzycki $\mathrm{S}_{1}$ et al. Do $\mathrm{CHA}_{2} \mathrm{~S}_{2}$ $D_{2}$-VASc and HAS-BLED scores influence 'real-world' anticoagulation management in atrial fibrillation? 1556 patient registry from the reference cardiology centre. Pharmacoepidemiol Drug Saf 2015;24:1297-1303.

63. Hori M, Matsumoto $M$, Tanahashi N, Momomura S, Uchiyama S, Goto S, et al. Rivaroxaban vs. Warfarin in Japanese patients with atrial fibrillation: the J-ROCKET AF study. Circ J 2012;76: 2104-2111.

64. Tanigawa T, Kaneko M, Hashizume K, Kajikawa M, Ueda H, Tajiri $M$, et al. Model-based dose selection for phase III rivaroxaban study in Japanese patients with non-valvular atrial fibrillation. Drug Metab Pharmacokinet 2013;28:59-70.

65. Ruff CT, Giugliano RP, Braunwald E, Morrow DA, Murphy SA, Kuder JF, et al. Association between edoxaban dose, concentration, anti-factor xa activity, and outcomes: an analysis of data from the randomised, double-blind ENGAGE AF-TIMI 48 trial. Lancet 2015;385:2288-2295.

66. Koretsune Y, Yamashita T, Kimura T, Fukuzawa M, Abe K, Yasaka M. Short-term safety and plasma concentrations of edoxaban in Japanese patients with non-valvular atrial fibrillation and severe renal impairment. Circ J 2015;79:1486-1495.

67. Graham DJ, Reichman ME, Wernecke M, Zhang R, Southworth MR, Levenson $M$, et al. Cardiovascular, bleeding, and mortality risks in elderly medicare patients treated with dabigatran or warfarin for nonvalvular atrial fibrillation. Circulation 2015; 131:157-164

68. Chan YH, Yen KC, See LC, Chang SH, Wu LS, Lee HF, et al. Cardiovascular, bleeding, and mortality risks of dabigatran in Asians with nonvalvular atrial fibrillation. Stroke 2016;47:441449.

69. Ogawa S, Aonuma K, Tse HF, Huang D, Huang JK, Kalman J, et al. The APHRS's 2013 statement on antithrombotic therapy of patients with nonvalvularatrial fibrillation. J Arrhythmia 2013; 29:190-200.

70. Jung KH, Yu KH, Kim YD, Park JM, Hong KS, Rha JH, et al. Antithrombotic management of patients with nonvalvular atrial fibrillation and ischemic stroke or transient ischemic attack: executive summary of the Korean clinical practice guidelines for stroke. J Stroke 2015;17:210-215. 